\title{
Demandas do trabalho e controle: implicações em unidades de terapia intensiva pediátrica e neonatal
}

\author{
Demands and control of work: implications in pediatric and neonatal intensive care units
}

Demandas y control del trabajo: implicaciones en las unidades de tratamiento intensivo pediatrico y neonatal

\section{Monalisa de Cássia Fogaça', Werther Brunow de Carvalho', Luiz Antônio Nogueira-Martins'}

'Universidade Federal de São Paulo. São Paulo, SP

Submissão: 16/11/2008

Aprovação: 18/05/2010

\section{RESUMO}

Comparar a demanda e o controle sobre trabalho de médicos e enfermeiros Que trabalham em unidades de tratamento intensivo pediátrica e neonatal. Estudo transversal com 37 médicos e 20 enfermeiros. Utilizou-se o Job Content Questionnarie. Médicos da UTI Neonatal diferem em suporte social recebido do supervisor $(p=0,0$ I) em relação aos da UTI Pediátrica. Enfermeiros da UTI Neonatal apresentam insegurança no trabalho $(p=0,05)$. Médicos e enfermeiros da UTI Pediátrica diferem em controle sobre o trabalho; demanda psicológica do trabalho; esforço físico e suporte do supervisor $(\mathrm{p}<0,05)$. Médicos e enfermeiros da UTI Neonatal apresentam diferenças estatísticas em controle sobre o trabalho; esforço físico e suporte do supervisor $(p<0,05)$. O ambiente de trabalho nas UTI's apresenta alta demanda e baixo controle sobre o trabalho.

Descritores: Equipe de enfermagem; Estresse; Unidades de terapia intensiva; Médicos.

\section{ABSTRACT}

To compare the demand and control over work of physicians and nurses working in pediatric and neonatal critical care units. Crosssectional study with 37 doctors and 20 nurses. We used the Job Content Questionnarie. Physicians from the ICU Neonatal differ in social support received from the supervisor $(p=0.01)$ compared to the ICU Pediatric. ICU nurses of the Neonatal present job insecurity $(p=0.05)$. Physicians and nurses from the ICU Pediatric differ in control over the work, psychological demands of work, physical effort and support of the supervisor $(\mathrm{p}<0.05)$. Physicians and nurses from the ICU Neonatal show statistical differences in control over the work, physical effort and support of the supervisor $(\mathrm{p}<0.05)$. The work environment in the ICU'S presents high demand and low control over the work.

Key words: Nursing team; Stress; Intensive care units; Physicians.

\section{RESUMEN}

Comparación de la demanda y control sobre el trabajo de los médicos y enfermeras Que trabajan en las unidades de tratamiento intensivo pediátrico y neonatal. Estudio transversal con 37 médicos y 20 enfermeras. Se utilizó el Job Content Questionnarie. Los médicos de la UCI Neonatal difieren en el apoyo social recibido desde el supervisor $(p=0,0 \mathrm{I})$ en comparacion com la UCI Pediátrica. Enfermeras de la UCI Neonatal actualmente la inseguridad en el empleo $(\mathrm{p}=0,05)$. Los médicos y enfermeras de la UCI Pediátrica difieren em el control sobre el trabajo, las exigencias psicológicas del trabajo, esfuerzo físico y el apoyo del supervisor $(p<0,05)$. Los médicos y enfermeras de la UCI Neonatal demostrar diferencias estadísticamente significativas en el control de la labor, esfuerzo físico y el apoyo del supervisor ( $p<0,05$ ). El entorno de trabajo en la UCI están en alta demanda y bajo el control de la obra.

Descriptores: Equipo de enfermería; Estrés; Unidades de terapia intensiva; Médicos.

AUTOR CORRESPONDENTE Monalisa de Cássia Fogaça. Rua Dr. Clemente Ferreira, I26, apto 24. CEP 095530-440. São Caetano do Sul, SP. E-mail: monalisa.cassia@uol.com.br 


\section{INTRODUÇÃO}

A relação entre o estresse ocupacional e a saúde mental do trabalhador vem sendo tema de várias pesquisas nos últimos anos, em nosso meio e em outros países, pois são preocupantes os índices de incapacitação temporária, absenteísmo, aposentadoria precoce e riscos à saúde associados à atividade laboral ${ }^{(1)}$.

Diversos estudos desenvolveram modelos teóricos a fim de avaliar a prevalência de distúrbios emocionais e disfunções profissionais no ambiente ocupacional e, identificar os fenômenos Que são comparáveis em múltiplos postos de trabalho e em grupos ocupacionais distintos $^{(2-4)}$.

Um dos modelos conceituais mais utilizados é o Modelo Demanda-Controle ${ }^{(2)}$, Que privilegia duas dimensões psicossociais no trabalho: o controle sobre o trabalho e a demanda advindo do trabalho. Karasek define Que a tensão no trabalho (job strain) ocorre Quando as demandas (exigências) do trabalho são altas e a margem de tomadas de decisões é baixa. Essa condição pode acarretar consequências nocivas à saúde física e psicológica.

O processo de trabalho nas UTIs demanda importantes atribuições, uma vez Que a gravidade e complexidade dos pacientes impõem a necessidade de lidar com equipamentos sofisticados, realizar avaliações clínicas constantes e procedimentos complexos, com tomada de decisões imediatas.

O estresse pode ser especialmente importante em Terapia Intensiva Pediátrica e Neonatal. Médicos e enfermeiros têm sido identificados como um grupo de extensiva atividade laboral, com demandas físicas e emocionais, ocasionada pelas implicações de suas atividades ocupacionais ${ }^{(5-19)}$.

$\mathrm{Na}$ maioria das vezes, o médico lida com a complexa relação entre os diversos profissionais, combinando funções administrativas com a de ensino e assistência, enQuanto o enfermeiro coordena os serviços auxiliares e de apoio à assistência. Assim, enQuanto o médico fica mais sujeito a uma sobrecarga emocional ${ }^{(20)}$, o enfermeiro parece sofrer mais com a sobrecarga física do trabalho ${ }^{(21-24)}$.

Diante do exposto acima, o principal objetivo desse estudo é comparar a demanda e o controle sobre trabalho de médicos que trabalham em UTI Pediátrica e Neonatal, e, também, entre enfermeiros que trabalham nas mesmas unidades. O estudo também pretende avaliar se há diferença entre o controle sobre o trabalho e a demanda psicológica na mesma categoria profissional, mas diferindo de acordo com a unidade de terapia intensiva.

\section{MÉTODOS}

Realizou-se um estudo transversal, Que incluiu médicos e enfermeiros, Que trabalhavam em UTI Pediátrica (35) e Neonatal (22), na Universidade Federal de São Paulo/Escola Paulista de Medicina (UNIFESP/EPM). Foram avaliados ao todo 25 médicos e 10 enfermeiros da UTI Pediátrica e 12 médicos e 10 enfermeiros da UTI Neonatal, constituindo-se a amostra total de 57 profissionais, Que aceitaram participar espontaneamente do estudo. O critério de inclusão consistia em ser médico ou enfermeiro contratado para trabalho na UTI e médico-residente cursando o estágio de UTI.

O protocolo de pesquisa foi aprovado pelo Comitê de Ética e Pesquisa da UNIFESP/EPM (no 1604/04) e todos os participantes assinaram o termo de Consentimento Livre e Esclarecido.

Utilizou-se o Job Content Questionnarie (JCQ) com 49 Questões, elaborado por Robert Karasek (Universidade de Massachusetts, Lowell, 1979) e traduzido e adaptado no Brasil por Tânia M. Araújo - Núcleo de Epidemiologia (Universidade de Feira de Santana, Bahia, Brasil, 2001).

As variáveis analisadas foram: controle sobre o trabalho (autoridade decisória e autoridade decisória no nível macro); demandas psicológicas do trabalho; esforço físico; carga isométrica física; demandas físicas do trabalho; insegurança no emprego; suporte social proveniente do supervisor; suporte social proveniente dos colegas de trabalho. A medida de resposta de cada item é avaliada em 4 níveis: 1 = discordo fortemente, 2 = discordo, $3=$ concordo e $4=$ concordo fortemente. O coeficiente de Alfa Cronbach geralmente aceito para mulher é 0,73 e para homem é $0,74^{(2)}$.

As comparações entre unidades de trabalho (UTI Pediátrica e UTI Neonatal) e categoria profissional (Médicos e Enfermeiros) foram estudadas utilizando o teste Mann-Whitney $\mathrm{U}$.

\section{RESULTADOS}

Os médicos eram predominantemente do sexo feminino (76 \%) com média etária de 34,70 $\pm 7,11$ anos, e trabalhavam em UTI, em média há $7,17 \pm 6,89$ anos. Os enfermeiros eram predominantemente do sexo feminino (95\%), com média etária de $31,55 \pm 6,37$ anos, e trabalhavam na UTI, em média, há 5,85 \pm 4,40 anos.

$\mathrm{Na}$ análise do ICQ pode-se dizer Que médicos da UTI Pediátrica comparados aos da UTI Neonatal apresentam valores médios superiores nas seguintes variáveis estudadas: controle sobre o trabalho - autoridade decisória $(35,68 \pm 5,88)(p=0,19)$; esforço físico $(6,32 \pm 1,46),(p=0,22)$; carga isométrica física $(5,00 \pm$ $1,29),(p=0,62)$ e suporte social proveniente de colegas de trabalho $(11,84 \pm 0,98),(p=0,29)$. Nas demais variáveis: demanda psicológica do trabalho $(43,58 \pm 4,89),(p=0,92)$; insegurança no trabalho $(6,25 \pm 2,86)$, $(p=0,43)$, médicos da UTI Neonatal apresentaram valores médios superiores, comparados aos da UTI Pediátrica, além de suporte social recebido do supervisor (14,83 $\pm 1,16),(p=0,01)$, com diferença estatisticamente significante (tabela 2).

Em relação à análise realizada com os enfermeiros Que compõe a UTI Pediátrica, obtiveram-se valores médios superiores nas seguintes variáveis, comparados com os da UTI Neonatal: controle sobre o trabalho - autoridade decisória $(33,20 \pm 4,64),(p=0,83)$; esforço físico $(7,40 \pm 1,40)$, $(p=0,09)$; e suporte provenientes dos colegas $(11,50 \pm 0,71),(p=0,11)$, sem diferença estatisticamente significante. Enfermeiros da UTI Neonatal apresentaram valor com significância estatística na variável insegurança no trabalho $(5,80 \pm 2,44),(p=0,05)$. Nas demais variáveis: demanda psicológica do trabalho $(35,70 \pm 7,47)$, $(p=0,93)$; carga isométrica física $(4,90 \pm 0,99),(p=0,26)$ e suporte social proveniente do supervisor $(11,10 \pm 5,72)$, $(p=0,68)$, nenhuma diferença estatística foi encontrada, Quando comparados com enfermeiros da UTI Pediátrica (tabela 2).

Quando comparadas categorias profissionais nas diferentes unidades de trabalho pode-se dizer Que médicos e enfermeiros da 
Tabela 1. Demanda e controle sobre o trabalho entre médicos e enfermeiros de UTI pediátrica e neonatal.

\begin{tabular}{|c|c|c|c|c|c|c|c|c|}
\hline \multirow[t]{2}{*}{ Questionário sobre Conteúdo do Trabalho } & \multicolumn{3}{|c|}{ Médicos } & \multicolumn{3}{|c|}{ Enfermeiros } & \multicolumn{2}{|c|}{$\begin{array}{c}\text { Médico } \\
\text { vs. } \\
\text { Enfermeiro }\end{array}$} \\
\hline & PED & $N E O$ & $p$ & PED & $N E O$ & $p$ & PED (p) & $N E O(p)$ \\
\hline Controle sobre o trabalho: decisão autoritária & 35,68 & 33,33 & 0,19 & 33,20 & 31,60 & 0,83 & 0,02 & 0,02 \\
\hline Demanda psicológica do trabalho & 34,48 & 43,58 & 0,92 & 32,20 & 35,70 & 0,93 & 0,03 & 0,11 \\
\hline Esforço físico & 6,32 & 5,75 & 0,22 & 7,40 & 7,30 & 0,09 & 0,02 & 0,05 \\
\hline Carga isométrica física & 5,00 & 4,67 & 0,62 & 4,80 & 4,90 & 0,26 & 0,73 & 0,24 \\
\hline Demandas físicas do trabalho & 11,32 & 10,42 & 0,33 & 12,20 & 12,20 & 0,62 & 0,13 & 0,07 \\
\hline Insegurança no trabalho & 5,72 & 6,25 & 0,43 & 5,30 & 5,80 & 0,05 & 0,47 & 0,89 \\
\hline Suporte Social do Supervisor & 10,68 & 14,83 & 0,01 & 10,90 & 11,10 & 0,06 & 0,04 & 0,02 \\
\hline Suporte proveniente dos colegas & 11,84 & 11,42 & 0,29 & 11,50 & 11,00 & 0,11 & 0,30 & 0,31 \\
\hline
\end{tabular}

$P E D=$ unidade de terapia intensiva pediátrica $N E O=$ unidade de terapia intensiva neonatal

UTI Pediátrica diferem nas seguintes variáveis, com diferenças estatísticas significantes: controle sobre o trabalho - autoridade decisória $(p=0,02)$; demanda psicológica do trabalho $(p=0,03)$; esforço físico $(p=0,02)$ e suporte proveniente do supervisor $(p=0,04)$ (Tabela 2).

Em relação a médicos e enfermeiros Que compõe a UTI Neonatal, diferenças estatísticas significantes foram encontradas nas variáveis: controle sobre o trabalho - autoridade decisória $(p=0,02)$; esforço físico $(p=0,05)$ e suporte proveniente do supervisor $(p=0,02)$.

\section{DISCUSSÃO}

A utilização do instrumento JCQ, para avaliar o estresse ocupacional de enfermeiros, tem sido recurso metodológico em alguns estudos ${ }^{(25-27)}$.

Nestes estudos, pode-se verificar Que um dos pressupostos adotado por Karasek ${ }^{(2,28)}$, Que a tensão psicológica sofrida no trabalho, ou seja, Quando a as demandas são altas e o trabalhador tem baixa autonomia, como conseqüência gerando tensão no ambiente organizacional, é observada, assim como na presente pesQuisa.

Em nosso estudo encontramos valores estatísticos significantes na variável suporte social proveniente do supervisor, presentes em médicos da UTI Neonatal. A possibilidade de apoio social diminui a tensão psicológica, Que pode depender do grau de interação social e emocional e da confiança Que se estabelece entre os membros da equipe e o supervisor ${ }^{(2,28)}$.

Em contrapartida observou-se Que enfermeiros da UTI Neonatal apresentam valores estatísticos significantes na variável insegurança no trabalho. Este fato é descrito por Karasek ${ }^{(2,28)}$, como alta exigência e baixo controle, somado a um baixo apoio social, com maiores riscos para o desenvolvimento de patologias físicas e mentais.

Quando comparamos médicos e enfermeiros da UTI Pediátrica, observamos diferenças estatísticas significativas em relação às variáveis: controle sobre o trabalho, demanda psicológica do trabalho, esforço físico e suporte social proveniente do supervisor. Estas diferenças retratam um ambiente organizacional Que apresenta grande tensão, ou seja, a autonomia dos profissionais para tomar decisões (controle sobre o trabalho) e a alta demanda, produz desmotivação e dificuldade no aprendizado em novas tarefas, além de reduzida habilidade para gerenciar situações organizações ${ }^{(2,28)}$.
Da mesma forma, podemos afirmar em relação às comparações entre médicos e enfermeiros da UTI Neonatal, onde apresentam diferenças significativas nas variáveis: controle sobre o trabalho, esforço físico e suporte social provenientes do supervisor.

No estudo de Rijk(25), apesar de enfermeiros de UTI, apresentarem alto controle sobre o trabalho, tendia a uma maior exaustão emocional, em virtude da demanda advinda do mesmo; o Que corrobora nossa pesquisa.

Apesar de não avaliarmos as diferenças entre gêneros, LaMontagne $^{(29)}$ e Muhonen $^{(30)}$, demonstraram Que o controle sobre o trabalho e demandas advindas do mesmo, são vivenciadas de forma diferente por homens e mulheres. No primeiro estudo a prevalência de tensão no trabalho é maior em mulheres, e as altas demandas têm um efeito direto sobre a saúde da mulher, enquanto Que no sexo masculino, influência as relações sociais, fato observado no segundo estudo.

A maioria da população por nós avaliada faz parte do universo feminino, e nossos resultados apresentam ambientes organizacionais marcados pelo estresse e altas demandas, apontando baixa autonomia no trabalho vivenciada pela maioria dos sujeitos analisados.

Vale ressaltar Que estudos de corte transversal caracteristicamente apresentam dois tipos de limitações: só incluem aqueles indivíduos Que "sobreviveram" à doença e, por coletarem simultaneamente dados de exposição e da doença, têm dificuldade para estabelecer uma relação de causalidade entre ambos. Desta forma, não podemos deixar de considerar as possibilidades de havermos perdido informações daqueles Que: (1) não devolveram os Questionários; ou (2) por alguma razão, estavam ausentes do trabalho na época da coleta de dados.

O tipo de amostragem de conveniência utilizado pode ter comprometido a representatividade.

\section{CONCLUSÕES}

Apesar do número limitado de participantes, o estudo tem importantes implicações para futuras pesQuisas com o instrumento ICQ em UTIs, na tentativa de verificar fatores ocupacionais, bem como comparar dados sócio-demográficos com o controle e demanda no trabalho, além de apoio social advindo do trabalho, 
visando instrumentar profissionais de áreas afins, Quanto à detecção e redução de fatores de riscos ocupacionais, bem como direcionar o trabalhador para redução de formas inadequadas de lidar com as exigências advindas do trabalho.

\section{REFERÊNCIAS}

1. Vieira LC, Guimarães LAM, Grubits S. O estresse ocupacional em enfermeiros. In: Guimarães LAM, Grubits S, organizadoras. Saúde mental e trabalho. $3^{\text {a }}$ ed. São Paulo: Casa do Psicólogo; 2003. p. 169-85.

2. Karasek RA. Job demand, job decision latitude, and mental strain: implication for job redesign. Adm Sci Q 1979; 24: 285-308.

3. Maslach C, Jackson SE. The measurement of experienced burnout. J Occup Behavior, 1981; 2:99-113.

4. Siegrist J. The model of effort-reward imbalance: theorical backgroud: information and documentation - bibliographic references [online]. 1999. Disponível em: http://www.uniduesseldorf.de/www/workstress/htm.

5. Oehler IM, Davidson MG, Starr LE, Lee DA. Burnout, job stress, anxiety, and perceived social support in neonatal nurses. Hearth Lung 1991; 20 (5 Pt 1): 500-5.

6. Oehler JM, Davidson MG. Job stress and burnout in acute and nonacute pediatric nurse. Am I Crit Care, 1992; I (2):81-90.

7. Benica SW, Longo CB, Barnsteiner JH. Perceptions and significance of patient deaths for pediatric critical care nurses. Crit Care Nurse 1992; 12(3): 72-5.

8. Downey V, Bengiamin M, Heuer L, Juhl N. Dying babies and associated stress in NICU nurses. Neo Netw 1995; $14(1): 41-46$.

9. Oates RK e Oates P. Stress and mental health in neonatal intensive care units. Arch Dis Child 1995;72: F107-FI 10.

10. Oates PR, Oates RK. Stress and work relationships in the neonatal intensive care unit: are they worse than in the wards? I Paediatr Child Health 1996; 32 (1):57-59.

11. Fields AL, Cuerdon TT, Brasseux CO, Getson PR, Thompson $\mathrm{AE}$, Orlowski JP et al. Physician burnout in pediatric care medicine. Crit Care Med 1995; 23(8): 1425-29.

12. Gibbons C, Geller S, Glatz E. Biomedical equipment in the neonatal intensive care unit: Is it a stressor? | Perinat Neonatal Nurs, 1998;12(3):67-73.

13. Fischer JE, Calame A, Detting AC, Zeier H, Fanconi S. Experience and endocrine stress responses in neonatal and pediatric critical care nurses and physicians. Crit Care Med 2000; 28(9): 3281-8.

14. Arriortua AB, Cid JL, Álvarez AC, Escribano V, García LN, Carvavilla EP. Situación de burnout de los pediatras intensivistas españoles. An Esp Pediatr 2000;52: 41 8-23.

15. Bratt MM, Broome M, Kelber S, Lostocco L. Influence of stress and nursing leadership on job satisfaction of pediatric intensive care unit nurses. Am I Crit Care 2000; 9:307-17.

16. Morrison WE, Haas EC, Shaffner DH, Garrett ES. Noise, stress, and annoyance in pediatric intensive care unit. Crit Care Med,
2003;31(1):113-119.

17. De Martino MMF e Misko MD. Estados emocionais de enfermeiros no desempenho profissional em unidades críticas. Rev Esc Enferm USP 2004; 38(2): 161-7.

18. Cronevist A, Lützen K, Nyström M. Nurses' lived experiences of moral stress support in the intensive care context. I Nurs Manag 2006; I 4: 405-I3.

19. Hays MA, All AC, Mannahan C, Cuaderes E, Wallace D. Reported stressors and ways of coping utilized by intensive care unit nurses. Dimens Crit Care Nurs, 2006; 25(4): 185-193.

20. Bühler KE e Land T. Burnout and personality in intensive care: an empirical study. Hosp Top, 2003;8 I (4):5- 12.

21 . Ferrareze MVG, Ferreira V, Carvalho AMP. Percepção do estresse entre enfermeiros Que atuam em Terapia Intensiva. Acta Paul Enferm 2006; 19(3): 310-15.

22. Spindola T, Santos RS. O trabalho na enfermagem e seu significado para as profissionais. Rev Bras Enferm 2005; 58(2): 156-60.

23. Montanholi LL, Tavares DMS, Oliveira GR. Estresse: fatores de risco no trabalho do enfermeiro hospitalar. Rev Bras Enferm 2006; 59(5): 66I-5.

24. Magnago TSBS, Lisboa MTL, Souza IEO, Moreira MC. Distúrbios músculo-esqueléticos em trabalhadores de enfermagem: associação com condições de trabalho. Rev Bras Enferm 2007; 60(6): 701-5.

25. Rijk AE, Le Blanc PM, Schaufeli WB. Active coping and need for control as moderators of the job demand-control model: effects on burnout. I Occup Org Psych 1998; 71: 1-18.

26. Tzeng $\mathrm{H}$. Nurses self-assessment of their nursing competencies, job demands and job performance in the Taiwan hospital system. Intern I Nurs Studies 2004; 41: 487-96.

27. Hall D S. The relationship between supervisor support and registered nurse outcomes in nursing care units. Nurs Admin Q 2007; 3 I (1): 68-80.

28. Karasek RA, Brisson C, Kawakami N, Houtman I, Bongers P, Amicks B. The Job Content Questionnarie (JCQ): an instrument for internationally comparative assessments of psychosocial job characteristics. I Occup Health Psychol 1998; 3(4): 322-35.

29. LaMontagne AD, Keegel T, Vallance D, Ostry A, Wolfe R. Job strain - Attributable depression in a sample of working Australians: Assessing the contribution to health inequalities. BMC Public Health 2008; 8: 181.

30. Muhomen T, Torkelson E. The demand-control-support model and health among women and men in similar occupations. I Behav Med 2003; 26(6): 601-13. 